\title{
Clinical significance of antibodies against neutrophils in patients with inflammatory bowel disease and primary sclerosing cholangitis
}

\author{
F Seibold, P Weber, R Klein, P A Berg, K H Wiedmann
}

\begin{abstract}
The presence of perinuclear antibodies against neutrophils (pANCA) has been detected recently in sera of patients with inflammatory bowel disease and primary sclerosing cholangitis. In order to evaluate their clinical significance, sera from 126 patients with inflammatory bowel disease ( 80 Crohn's disease and 46 ulcerative colitis and 22 patients with primary sclerosing cholangitis were examined for pANCA by indirect immunofluorescence on liver sections and cytocentrifuge slides of neutrophils and by immunoblot. Perinuclear antibodies against neutrophils were found in $83 \%$ of patients with ulcerative colitis in $88 \%$ of patients with primary sclerosing cholangitis and inflammatory bowel disease, in $40 \%$ of patients with primary sclerosing cholangitis but without inflammatory bowel disease, and in $25 \%$ of patients with Crohn's disease using the immunofluorescence test. Titres of pANCA ranged from 1:10 to 1:1000 in ulcerative colitis and primary sclerosing cholangitis (median 1:100), whereas in Crohn's disease only four patients had titres of more than 1:10. The occurrence of pANCA did not correlate with clinical activity of Crohn's disease and primary sclerosing cholangitis whereas in ulcerative colitis high titres of PANCA were found mainly in active disease. Using an immunoblot system with sonified neutrophils as antigen, $82 \%$ of sera from patients with primary sclerosing cholangitis reacted with up to five different determinants, whereas only $12 \%$ of sera from patients with Crohn's disease and $11 \%$ of sera with ulcerative colitis detected one of the determinants, suggesting different antigens involved in PANCA reaction.
\end{abstract}

Inflammatory bowel diseases are associated with abnormal liver function tests in up to $95 \%$ of affected patients' and with primary sclerosing cholangitis in 1 to $4 \% .^{2}$ The aetiology and the pathogenesis of primary sclerosing cholangitis and inflammatory bowel disease is still unknown. In primary sclerosing cholangitis a high association with HLA-B8/DR3 and especially with DRw52a had been reported, ${ }^{3+}$ whereas in inflammatory bowel disease no strong association with HLA antigens could be found. ${ }^{5}$ Disturbed functions of the immune system, cellular and humoral, have been shown in inflammatory bowel disease and several associated autoantibodies have been found. The pathogenetic relevance of these antibodies is unclear, however, although some of them may be of diagnostic relevance. ${ }^{6}$ Thus, antibodies against exocrine pancreas juice could be specifically detected in Crohn's disease and antibodies against goblet cells in ulcerative colitis. ${ }^{78}$ In primary sclerosing cholangitis a panel of various antibodies has been described, ${ }^{9}$ but their pathogenetic or diagnostic relevance is not yet clear. Investigations for antibodies occurring in sera of patients with inflammatory bowel disease and primary sclerosing cholangitis on liver sections showed an antibody reacting exclusively with nuclei of cells infiltrating the portal tract. First, Chapman $e t$ $a l^{10}$ reported of this antibody considering a reaction against proliferating bile duct epithelia. In double labelling studies with monoclonal antibodies, however, Snook et $a l^{11}$ and $\mathrm{we}^{12}$ could show that this antibody is not directed against bile duct epithelia, but rather against the neutrophils distributed in liver sections. Because of the reactivity of the antibody mainly in the perinuclear area of neutrophils, the antibody was called pANCA. ${ }^{13}$

In this study, sera of patients with inflammatory bowel disease and primary sclerosing cholangitis were tested on cryostat sections of different organs and on neutrophils by immunofluorescence and immunoblotting, to evaluate the specificity of antibodies against neutrophils in respect to clinical features.

\section{Methods}

\section{PATIENTS}

Sera of 126 patients with inflammatory bowel disease were analysed in this study. The diagnosis of Crohn's disease was established in 80 of the patients ( 39 females, 41 males) by endoscopic, histological, and clinical criteria. ${ }^{14}$ The age of the patients ranged from 18 to 60 years. The mean duration of disease was eight years (range: 12 weeks-31 years). Thirty one (39\%) of the patients were on treatment with steroids at the time of blood sampling. The following segments of intestine were involved: colon exclusively $(\mathrm{n}=$ $16,20 \%)$, colon and ileum ( $n=49,61 \%$; of these, $\mathrm{n}=$ three also with involvement of stomach and duodenum), small intestine without colon involvement $(n=15,19 \%)$. Extraintestinal manifestations (erythema nodosum, pyoderma gangrenosum, iridocyclitis, oligoarthritis and sacroileitis) were found in $18(20 \%)$ of the patients. Fistula were detected in six patients. For grading of the disease activity, the Crohn's Disease Activity Index (CDAI) was used. ${ }^{15}$

In 46 patients $(27$ female and 19 male) ulcerative colitis was diagnosed according to the criteria of Truelove and Witts. ${ }^{16}$ The age ranged 
from 23 to 77 years. Twenty five patients had a left side colitis and 21 a pancolitis. The mean duration of disease was 10 years (range: three months-32 years). Activity was graded according to Truelove and Witts. ${ }^{16}$ Seven patients had severe disease, the remaining were classified as moderate or in remission. Twelve patients were being treated with corticosteroids at the time of blood sampling. In four patients a proctocolectomy had been performed. Sera from 22 patients with primary sclerosing cholangitis were also included in this study (20 males, two females). The diagnosis of primary sclerosing cholangitis was established by clinical, endoscopic and histological criteria. ${ }^{17}$ Seventeen of these patients had inflammatory bowel disease in addition to primary sclerosing cholangitis (13 patients with ulcerative colitis four patients with Crohn's disease). These 17 patients were not included in the inflammatory bowel disease group. The age ranged from 20 to 52 years. The mean duration of the disease was six years. In two patients a liver transplantation had been carried out.

In addition, control sera from 102 other patients were tested: 25 with primary biliary neutrophils with pANCA serum and Wegener serum (Fig 3). cirrhosis, 15 with autoimmune chronic active hepatitis, 15 with viral hepatitis (B and C), six with Wegener's granulomatosis, 25 with defined collagen disorders, four with secondary biliary cirrhosis and 30 sera from healthy blood donors with different blood groups. All sera were stored at $-20^{\circ} \mathrm{C}$.

\section{ISOLATION AND PREPARATION OF NEUTROPHILS}

Heparinised blood of healthy volunteers (blood group () was layered onto Mono-Poly Resolving medium (Flow Laboratories) and centrifuged at $560 \mathrm{~g}$ for 30 minutes. The polymorph layer was carefully drawn off and washed in phosphate buffered saline. Cytocentrifuge slides were fixed with $95 \%$ ethanol at $4^{\circ} \mathrm{C}$ for four minutes and were stored at $-70^{\circ} \mathrm{C}$ until use.

\section{IMMUNOFLUORESCENCE TECHNIQUE}

Slides were incubated for 40 minutes in a moist chamber with $50 \mu \mathrm{l}$ serum samples (diluted 1:10 in phosphate buffered saline). After careful washing, the slides were incubated for a further 30 minutes with polyvalent fluorescein conjugated sheep antihuman immunoglobulin (Wellcome, diluted 1:20 in phosphate buffered saline) at ambient temperature. Subsequently, after washing again, the slides were embedded in Immuno-Mount (Shandon) and analysed in a Leitz fluorescence microscope (HDO $200 \mathrm{~W}$ mercury lamp with filter combination I2, mirror RKP 510).

Immunofluorescence and an alternative immunoperoxidase technique were done on cryostat sections of human liver, liver from patients with bile duct obstruction and consecutive bile duct proliferation, human colon, and human pancreas. When the peroxidase technique was carried out, a peroxidase conjugated polyvalent antihuman immunoglobulin from sheep (Wellcome, diluted 1:20 in phosphate buffered saline was used after serum incubation. The peroxidase reaction was developed by incubation with $0 \cdot 1 \%$ $3^{\prime}-3$ 'diaminobenzidine-tetrahydrochloride and $0.003 \%$ peroxide for 10 minutes. Sections were counterstained with Mayer's haemalum (Merck) for 30 seconds and mounted in Kaiser's glyceringelatine (Merck). All slides were coded and read by two independent investigators.

TABLE I Incidence of $P A N C A$ (perinuclear antineutrophil cytoplasmic antibodies) and cANCA (classical anti-

neutrophil cytoplasmic antibodies) in patients with various diseases tested in immunofluorescence

\begin{tabular}{|c|c|c|c|}
\hline Disease & Sera $(n)$ & PANCA & CANCA \\
\hline Crohn's disease & 80 & $20(25 \%)$ & 0 \\
\hline Ulcerative colitis & +6 & $38\left(83^{\prime \prime} w\right)$ & 0 \\
\hline Primary sclerosing cholangitis with & & & \\
\hline inflammatory bowel disease & 17 & $15\left(88^{\circ} \circ\right)$ & 0 \\
\hline $\begin{array}{l}\text { Primary sclerosing cholangitis } \\
\text { without inflammatory bowel } \\
\text { disease }\end{array}$ & & $2\left(+0^{\circ 1}\right.$ & () \\
\hline Primary biliary cirrhosis & 25 & $7(28 \%)$ & 0 \\
\hline Secondary biliary cirrhosis & 4 & 0 & 0 \\
\hline Autoimmune hepatitis & 15 & $5\left(33^{\prime \prime}(1)\right.$ & 0 \\
\hline Viral hepatitis & 15 & $1\left(7^{\prime \prime \prime}\right)$ & 0 \\
\hline Collagen disorders & 25 & 0 & 0 \\
\hline Wegener's disease & 6 & () & $6\left(100^{\prime \prime}\right)$ \\
\hline Healthỵ persons & 30 & 0 & 0 \\
\hline
\end{tabular}


Figure 2: Titres of pANCA in patients with Crohn's disease, ulcerative colitis and primary sclerosing cholangitis. (Three sera without occurrence of pANCA and two sera with titres of $1: 10$ were of patients with primary sclerosing cholangitis without inflammatory bowel disease.)

Figure 3: Positive immunofluorescence of neutrophils with Wegener serum.
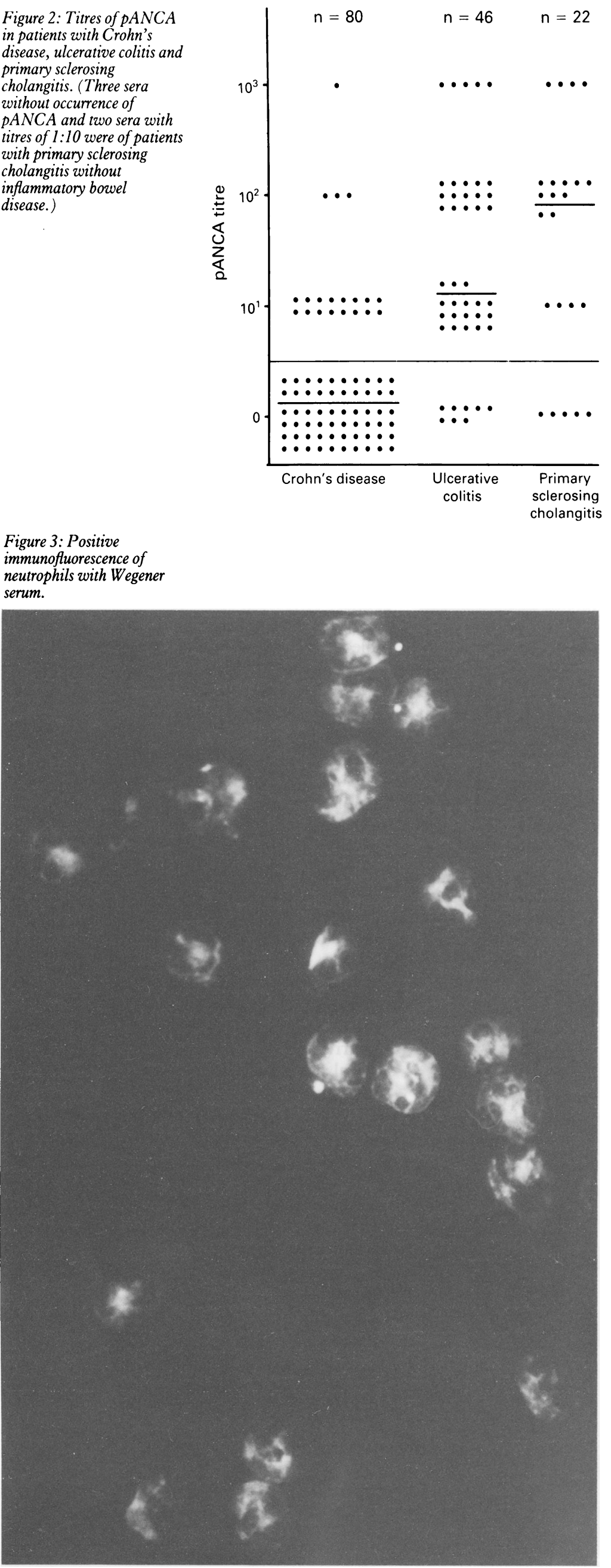

WESTERN BLOTTING WITH NEUTROPHILS

Sodium-dodecyl-sulphate electrophoresis with a $4.5 \%$ staking and a $10 \%$ running gel, and the following immunoblotting technique were carried out according to Laemmli ${ }^{18}$ and Towbin et al. ${ }^{19}$

Before applying to the gel, the neutrophil suspension was sonified for 60 seconds. After electrophoresis the gel was transferred onto immobilion sheets (Millipore) and incubated with phosphate buffered saline containing 3\% bovine serum albumin for two hours to diminish unspecific reactions. Then, the sera (1:50 diluted in phosphate buffered saline) were incubated for 45 minutes, washed in phosphate buffered saline, and exposed to peroxidase conjugated antihuman IgG and IgM (Dakopatts, 1:200 diluted in phosphate buffered saline) immunoglobulins for 30 minutes. The peroxidase reaction was developed by incubation with 3-amino-9-ethylcarbazole and peroxide.

\section{STATISTICAL ANALYSIS}

$\chi^{2}$ test or Fisher's exact test was used for statistical analysis.

\section{Results}

INCIDENCE OF PANCA IN PATIENTS WITH INFLAMMATORY BOWEL DISEASE AND PRIMARY SCLEROSING CHOLANGITIS

Thirty eight $(83 \%)$ of the sera of patients with ulcerative colitis showed a perinuclear fluorescence on cytocentrifuge slides of neutrophils (Fig 1, Table I) with titres ranging from $1: 10$ to $1: 1000$ (Fig 2). In Crohn's disease, pANCA occurred in $20(25 \%)$ of the patients, but only four of them had titres greater than 1:10 (Fig 2). The antibodies found in patients with inflammatory bowel disease, were all of $\operatorname{IgG}$ type. In addition about one third of the patients had IgA and two patients IgM antibodies.

In patients with primary sclerosing cholangitis and associated inflammatory bowel disease, pANCA were found in $15(88 \%)$ of 17 patients, but in only two $(40 \%)$ of five patients with primary sclerosing cholangitis without inflammatory bowel disease. Titres of pANCA in primary sclerosing cholangitis with associated 
inflammatory bowel disease ranged from 1:10 to $1: 1000$ (median $1: 100$ ), while titres in patients without inflammatory bowel disease were only 1:10 (Fig 2). All patients with primary sclerosing cholangitis had IgG antibodies, additionally in five patients $\operatorname{IgA}$ antibodies and in three patients IgM antibodies were found.

\section{SPECIFICITY OF PPANCA}

None of the 25 sera from patients with collagen disorders had pANCA (Table I). In $7 \%$ of the patients with acute or chronic hepatitis $B$ and $C$, $28 \%$ of the patients with primary biliary cirrhosis, and in $33 \%$ of the patients with autoimmune chronic active hepatitis pANCA was found with titres ranging from $1: 10$ to $1: 1000$. In all 30 sera of healthy blood donors and sera of the four patients with secondary biliary cirrhosis pANCA were not detectable.

Cytoplasmic staining of neutrophils was found in all sera of the six patients with Wegener's granulomatosis (Fig 3), clearly contrasting with the pattern of pANCA (Table I). Results in

Figure 4: Positive of neutrophils in liver section by $p A N C A$ positive serum.

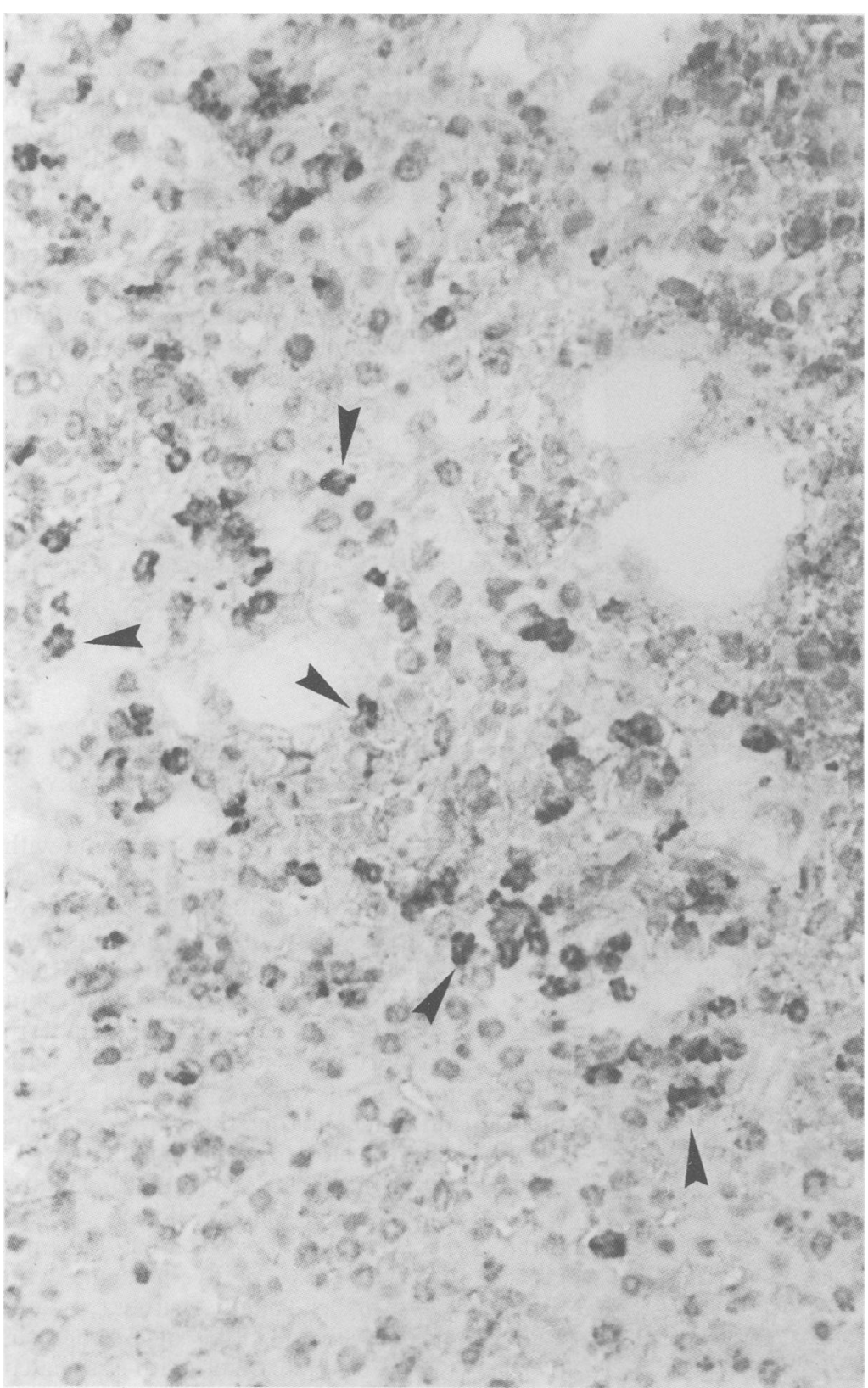

testing pANCA were not influenced when neutrophils from subjects with different blood groups were used.

\section{PANCA ON OBSTRUCTED LIVER}

On liver sections, especially on obstructed liver, using an immunperoxidase technique, nuclear staining of cells was found in $10(22 \%)$ of the 46 patients with ulcerative colitis in one $(1 \%)$ of the Crohn's disease patients and in eight (36\%) of 22 patients with primary sclerosing cholangitis ( $\mathrm{Fig}$ 4). In double labelling studies using monoclonal antibodies, it could be shown that the cells with nuclear staining were granulocytic cells and not bile duct epithelia or hepatocytes. All sera positive for these antibodies found on liver sections were positive for pANCA on cytocentrifuge slides of neutrophils, as well.

\section{DETECTION OF PANCA IN IMMUNOBLOT}

To clarify whether pANCA in primary sclerosing cholangitis and in inflammatory bowel disease are identical, sera were tested in Western blot with sonified neutrophils. Eighty two per cent of primary sclerosing cholangitis sera detected one or more of five antigenic determinants of a molecular weight of $95,60,55,40,30 \mathrm{kD}$ (Fig 5). Twelve $(71 \%)$ of primary sclerosing cholangitis patients with associated inflammatory bowel disease reacted with the $60 \mathrm{kD}$ determinant. In primary sclerosing cholangitis patients without inflammatory bowel disease, however, a positive reaction in only one patient could be found in immunoblot (Table II). Although in patients with ulcerative colitis pANCA were detectable in $83 \%$ by immunofluorescence, only in $11 \%$ of sera a specific reaction was found in immunoblot. In Crohn's disease, 10 of $80(13 \%)$ sera of patients with Crohn's disease had antibodies against one of the five antigenic determinants.

\section{CORRELATION OF PANCA WITH CLINICAL}

FEATURES

Occurrence of pANCA did not correlate with clinical activity of Crohn's disease. In ulcerative colitis, however, high titres of pANCA (over

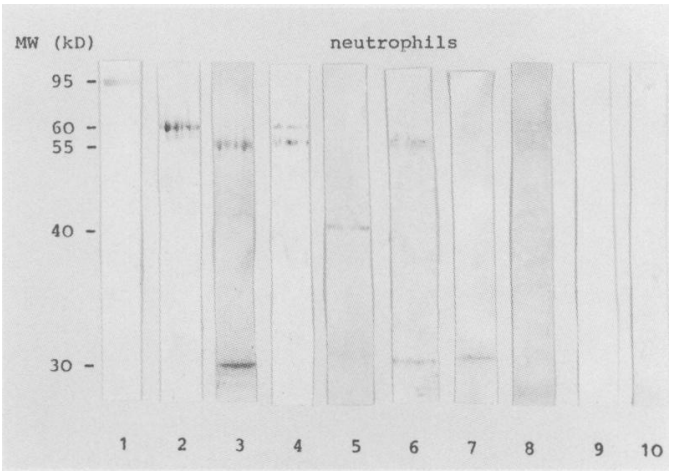

Figure 5: Immunoblotting with sonified neutrophils as antigen. Lane $1+2$ sera of patients with primary sclerosing cholangitis, lane 3-5: sera of patients with primary sclerosing cholangitis and ulcerative colitis, lane 6-8: sera of patients with ulcerative colitis ( $P A N C A$ positive in

immunofluorescence), lane 9: serum of patient with Crohn's disease ( $p A N C A$ positive in immunofluorescence), lane 10: serum of healthy person. 
$1: 10)$ were found mainly in active disease (significant for $\mathrm{p}<0.05$ ) and all of these patients needed steroid treatment. Four of the patients with pANCA had high titres before proctocolectomie. In long term observations persistence of titres over three years after surgery was noted. Titres of pANCA were not significantly influenced by steroid treatment. In ulcerative colitis and in Crohn's disease, there was no correlation of pANCA with age, sex of patients, the duration of disease, occurrence of extraintestinal manifestations or fistulas. Perinuclear antibodies against neutrophils were found in 15 of 65 patients (23\%) with Crohn's disease and colonic involvement and in four of 15 patients (27\%) with Crohn's disease involving small bowel exclusively. Titres over 1:10 were found in four patients with Crohn's disease: in three patients with colonic involvement and in one patient with small bowel involvement. Nineteen of 25 patients with ulcerative colitis and left side colitis had pANCA (76\%), in patients with pancolitis, pANCA was found in $81 \%$. In primary sclerosing cholangitis no correlation between titres of pANCA and aminotransferase, alanine aminotransferase, bilirubin, alkaline phosphatase or g-glutamyl transferase could be found. Two patients underwent liver transplantation and persistence of pANCA titres could be observed over two years after surgery.

COINCIDENCE OF PANCA WITH OTHER ANTIBODIES IN INFLAMMATORY BOWEL DISEASE

In Crohn's disease, there was no relationship between the occurrence of pANCA and the disease specific antibodies against pancreas found in $34 \%$ of patients. On the other hand, antibodies against goblet cells, specific for ulcerative colitis, occured in $14(30 \%)$ of the 46 patients with ulcerative colitis. In all of these 14 patients pANCA were also found, but titres of the two antibodies did not correlate (Table III).

\section{Discussion}

A new antibody type was evaluated which has recently been described in sera from patients

TABLE II Incidence of $p A N C A$ is immunofluorescence (IFL) in comparison with immunoblotting (reaction with one or more of the five antigens with a molecular weight of 30,40, $55,60,95 \mathrm{kD}$ )

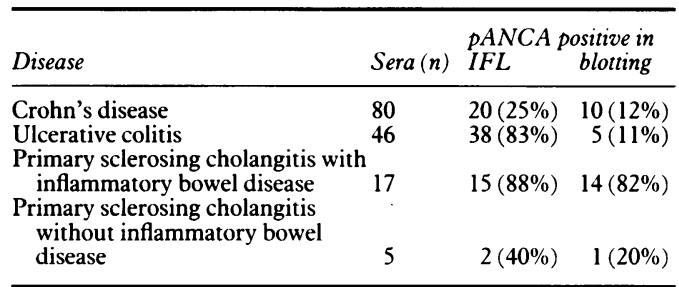

TABLE III Incidence of antibodies against neutrophils ( $p A N C A)$, antibodies against pancreas $(P A B)$ and intestina goblet cells $(G A B)$ in immunofluorescence as markers for the diagnosis of inflammatory bowel disease

\begin{tabular}{lllll}
\hline \multirow{5}{*}{ Positive for } \\
\cline { 2 - 5 } & Sera $(\mathrm{n})$ & pANCA & GAB & PAB \\
\hline Crohn's disease & 80 & $20(25 \%)$ & 0 & $27(34 \%)$ \\
Ulcerative colitis & 46 & $38(83 \%)$ & $14(30 \%)$ & 0 \\
\hline
\end{tabular}

with primary sclerosing cholangitis and inflammatory bowel disease. ${ }^{112021}$ This antibody reacted specifically with neutrophils displaying a perinuclear pattern by immunofluorescence and was therefore called pANCA. ${ }^{13}$ This is in contrast with the antibody occurring in Wegener's disease which shows a cytoplasmic granular fluorescence (classical antineutrophil cytoplasmatic antibody). ${ }^{13}$

In our series, similar to other authors, ${ }^{2021}$ pANCA were detected in the majority $(83 \%)$ of patients with ulcerative colitis, but also in Crohn's disease, albeit in a significant less prevalence $(25 \%)$ and lower titres. This is in contrast with Snook et al, ${ }^{\text {" }}$ using another staining method (immunperoxidase), who found no difference between the titres of pANCA in ulcerative colitis and Crohn's disease.

Furthermore, pANCA also occurred in $77 \%$ of patients with primary sclerosing cholangitis, which is interesting in so far as this bile duct disease is predominantly associated with ulcerative colitis and not with Crohn's disease. In patients with primary sclerosing cholangitis, pANCA titres tended to much higher values compared with patients with ulcerative colitis and Crohn's disease. In Crohn's disease, there is no relationship of pANCA whether Crohn's disease is extended to the colon or not, as was reported in another paper. ${ }^{20}$ Figures are small, however, and this important issue has to evaluated further. The relationship of presence of titre height of pANCA to disease activity was quite variable in our findings and is also inconsistent in the present literature. ${ }^{1120}$ Thus in ulcerative colitis, but not in Crohn's disease, titres correlated with disease activity, whereas in primary sclerosing cholangitis no correlation between clinical and laboratory findings was seen. Furthermore, our studies show that antibody titres were not influenced by treatment. It seems that other factors determine the expression of antibody production more profoundly, - that is, the underlying basic immunregulatory defect leading to the disease. This is supported by family studies, in which pANCA have been described in $21 \%$ of relatives of patients with ulcerative colitis, ${ }^{22}$ and by the fact that pANCA can persist for two or three years, after liver transplantation or proctocolectomy, respectively. The latter situation is similar to the findings of patients with primary biliary cirrhosis displaying persistent mitochondrial antibodies after liver transplantation..$^{23}$ So far, no recurrence of primary sclerosing cholangitis has been observed after liver transplantation.

In contrast with classical antineutrophil cytoplasmic antibodies which are very disease specific markers for patients with Wegener's granulomatosis, ${ }^{24} 25$ the specificity of pANCA found in inflammatory bowel disease or primary sclerosing cholangitis on immunofluorescence grounds is less well established. Perinuclear antibodies against neutrophils were also found in about $30 \%$ of patients with primary biliary cirrhosis and autoimmune hepatitis and in $7 \%$ of patients with chronic active or persistent hepatitis B or C. In other studies, 121 the prevalence in chronic liver diseases was even higher with findings of $52 \%$ (using immunoperoxidase tech- 
nique) and $63 \%$ respectively (using a fixed cell enzyme linked immunosorbent assay (ELISA). This may well be because of the different techniques applied for antibody detection and underlines the need for standardisation. Whereas obviously a considerable overlap between predominantly autoimmune liver diseases and inflammatory bowel disease and primary sclerosing cholangitis exists, pANCA did not occur either in secondary biliary cirrhosis, defined collagen disorders, or healthy persons.

Cross reactivity of an antibody with various diseases has always raised the question, whether the antigenic determinants recognised by the antibody are identical or different for various diseases. Preliminary results of immunoblot studies show that only about $12 \%$ of sera from patients with inflammatory bowel disease reacted with one or more of five determinants of a molecular weight of $30,40,55,60$, and $95 \mathrm{kD}$, whereas sera from patients with primary sclerosing cholangitis showed this reaction in $80 \%$. These findings indicate the existence of different antibodies against neutrophils in patients with inflammatory bowel disease and primary sclerosing cholangitis. Obviously, the antigen detected specifically by sera from patients with ulcerative colitis in immunofluorescence might be destroyed in the immunoblotting system, whereas the primary sclerosing cholangitis associated pANCA determinants are stable. Application of this technique to other disease groups might define the antigens involved in pANCA reactivity and is reported elsewhere..$^{26}$

So far, the nature of the antigens involved in primary sclerosing cholangitis and inflammatory bowel disease is not known. Classical antineutrophil cytoplasmic antibodies of Wegeners are reactive with an elastinolytic neutral serine proteinase. $^{2+}$ In earlier reports an antibody pattern similar to that seen in sera with primary sclerosing cholangitis or inflammatory bowel disease was found in sera from patients with rheumatoid arthritis, arteriitis, and glomerulonephritis. ${ }^{27} 28$ In these patients two different antibodies could be distinguished, one antibody displaying a perinuclear staining and reacting with myeloperoxidase and a second antibody showing a diffuse cytoplasmic fluorescence without reactivity against myeloperoxidase in ELISA. In our study, sera from primary sclerosing cholangitis and inflammatory bowel disease, tested on myeloperoxidase, were negative (results not shown).

The aetiology and possible pathogenetic role of pANCA remains unclear. Formation might be triggered by microorganisms or intestinal and biliary antigens in individuals with a genetic predisposition. ${ }^{+}$The predominant finding of pANCA, at least on immunofluorescence grounds, in ulcerative colitis and primary sclerosing cholangitis - clinically frequently associated diseases, raises the most interesting question where these antibodies reflect a common pathogenetic link between ulcerative colitis and primary sclerosing cholangitis.
1 Greenstein AJ, Janowitz HD, Sachar DB. The extraintestinal complications of Crohn's disease and ulcerative colitis. Medicine 1976; 55: 401-9.

2 LaRusso N, Wiesner R, Ludwig J, MacCarty R. Primary sclerosing cholangitis. N Eng F Med 1984; 310: 899-903.

3 Chapman RW, Varghese Z, Gaul R, Patel G, Kokinon N, Sherlock $S$. Association of primary sclerosing cholangitis with HLA-B8. Gut 1983; 24: 38-41.

4 Proghazka EJ, Terasaki PI, Park MS, Goldstein LI, Busuttil RW. Association of primary sclerosing cholangitis with HLA-DRw52s. N Eng 7 Med 1990; 322: 1842-44.

5 Biemond I, Burnham WR, d'Amaro J, Langman MJ. HLA-A and $-\mathrm{B}$ antigens in inflammatory bowel disease. Gut 1986; 27: $934-41$

6 Strober W, James SP. The immunologic basis of inflammatory bowel disease. 7 Clin Immunol 1986; 6: 415-32.

7 Stöcker W, Otte M, Ulrich S, Normann D, Finkbeiner H, Stöcker $\mathrm{K}$, et al. Autoimmunity to pancreatic juice in Crohn's disease. Scand $\mathcal{F}$ Gastroenterol 1987; 22 (suppl): Crohn's

8 Seibold F, Weber P, Jenss H, Wiedmann KH. Antibodies to a trypsin-sensitive pancreatic antigen in chronic inflammatory bowel disease: specific markers for a subgroup of patients with Crohn's disease. Gut 1991; 32: 1192-7.

9 Zauli D, Schrumpf E, Crespi C, Cassani F, Fausa O. Aadland E. An autoantibody profile in primary sclerosing cholangitis. F Hepatol 1987; 5: 14-8.

10 Chapman RW, Cottone M, Selby WS, Shepherd HA, Sherlock $S$, Jewell DP. Serum autoantibodies, ulcerative colitis and primary sclerosing cholangitis. Gut 1986; 27: 86-91.

11 Snook JA, Chapman RW, Fleming K, Jewell DP. Antineutrophil nuclear antibody in ulcerative colitis, Crohn's disease and primary sclerosing cholangitis. Clin Exp Immuno 1989; 76: 30-3.

12 Seibold F. Antikörper gegen Leber-, Darm- und Pankreasgewebe bei chronisch entzündlichen Darmerkrankungen. (Dissertation) Tübingen, 1990.

13 van der Woude FJ, Daha MR, van Es LA. The current status of neutrophil cytoplasmatic antibodies. Clin Exp Immunol 1989; 78: 143-8.

14 Malchow H, Ewe K, Brandes JW. European cooperative Crohn's disease study (ECCDS): Results of drug treatment. Gastroenterology 1984; 86: 249-66.

15 Best WJ, Bechtel J, Singleton J, Kern F. Development of a Crohn's disease activity index. Gastroenterology 1976; 70: 439-44.

16 Truelove SC, Witts LJ. Cortisone in ulcerative colitis: Final report of a therapeutic trial. $B M 71955 ; 2: 1041-8$.

17 Wiesner RH, LaRusso NF. Clinicopathologic features of the syndrome of primary sclerosing cholangitis. Gastroenterology 1980; 79: 200-6.

18 Laemmli U. Cleavage of structural proteins during assembly of the head of bacteriophage T4. Nature 1970; 27: 680-5

19 Towbin H, Staehelin T, Gordon J. Electrophoretic transfer of proteins from polyacrylamide gels to nitrocellulose sheets: Procedure and some applications. Proc Natl Acad Sci USA 1979; 76: 4350-4.

20 Saxon A, Shanahan F, Landers C, Ganz T, Targan S. A distinct subset of antineutrophil cytoplasmic antibodies is associated with inflammatory bowel disease. 7 Allergy Clin Immunol 1990; 66: 202-10.

21 Duerr RH, Targan SR, Landers CJ, LaRusso NF, Lindsay $\mathrm{KL}$, Wiesner RH, et al. Neutrophil cytoplasmic antibodies: $\mathrm{KL}$, Wiesner $\mathrm{RH}$, et al. Neutrophil cytoplasmic antibodies: A link between primary sclerosing cholangitis

22 Shanahan F, Duerr R, Rotter J, Yang H, Sutherland L, McElree C, et al. Neutrophil autoantibodies in ulcerative colitis: familial aggregation and genetic heterogeneity. Gastroenterology 1991; 100: A614

23 Haagsma ED, Manns M, Klein R, et al. Subtypes of antimitochondiral antibodies in primary biliary cirrhosis before and after orthotopic liver transplantation. Hepatology 1987; 7 129-33.

24 Lüdemann J, Utecht B, Gross W. Anti-neutrophil cytoplasm antibodies in Wegener's granulomatosis recognize an elastinolytic enzyme. $\mathcal{F}$ Exp Med 1990; 171: 357-62.

25 Nölle B, Specks U, Lüdemann J, Rohrbach MS, DeRemee RA, Gross WL. Anticytoplasmic autoantibodies: their immundiagnostic value in Wegener Granulomatosis. Ann Intern Med 1989; 111: 28-40.

26 Klein R, Eisenburg J, Weber P, Seibold F, Berg PA. Significance and specificity of antibodies against neutrophils Significance and specificity of antibodies against neutrophils
detected by western blotting for the serological diagnosis of detected by western blotting for the serological diagnosis of
primary sclerosing cholangitis. Hepathology 1991; 14: primary

27 Faber V, Elling P. Leucocyte-specific antinuclear factors in patients with Felty's syndrome, rheumatoid arthritis, systemic lupus erythematosus and other diseases. Acta Med Scand 1966; 179: 257-67.

28 Falk RJ, Jennette JC. Anti-neutrophil cystoplasmic autoantibodies with specificity for myeloperoxidase in patients with systemic vasculitis and idiopathic necrotizing and 1651-7. 